\title{
Emerging role of multikinase inhibitors for refractory thyroid cancer
}

\author{
This article was published in the following Dove Press journal: \\ Biologics: Targets and Therapy \\ 7 August 2012 \\ Number of times this article has been viewed
}

\section{Cesar A Perez' \\ Belisario A Arango' \\ Michel Velez' \\ Luis E Raez ${ }^{2}$ \\ Edgardo S Santos' \\ 'University of Miami Miller School of Medicine/Sylvester Comprehensive Cancer Center, Miami, USA; ${ }^{2}$ Memorial \\ Cancer Institute, Memorial Health \\ Care System, Hollywood, FL, USA}

Correspondence: Edgardo S Santos University of Miami Miller School of Medicine, Sylvester Comprehensive Cancer Center, I475 NW I2th Avenue, D8-4, Miami, FL 33।36, USA

Tel +I 3052436554

Fax + I 3052433289

Email esantos2@med.miami.edu
Abstract: Thyroid cancer incidence continues to increase, remaining the most common endocrine malignancy. The need for effective systemic therapies combined with high incidence of driver mutations and overexpression of molecular pathways make refractory thyroid cancer an ideal candidate for treatment with novel agents. Multikinase inhibitors have caused a paradigm shift in the treatment of patients with advanced iodine-refractory thyroid cancer. These agents have shown to be the most effective systemic therapy for this disease not only causing prolonged responses but also improving survival. The activity of these agents inhibiting several pathways simultaneously, such as rearranged during transfection protooncogene, mitogen-activated protein kinase, and angiogenesis, can probably explain the effectiveness in controlling the progression of this malignancy. Several of these agents are currently on clinical studies in patients with differentiated and medullary thyroid cancer and most of them are showing promising clinical activity. With the approval of vandetanib for the treatment of medullary thyroid cancer, a new era in the management of this disease has begun. The molecular rationale for the use of these drugs for thyroid cancer is discussed as well as their promising clinical results.

Keywords: axitinib, cabozantinib, lenvatinib, mitogen-activated protein kinase (MAPK), motesanib, pazopanib, thyroid cancer, vandetanib, vascular endothelial growth factor receptor-2 (VEGFR2)

\section{Introduction}

Thyroid cancer continues to be the most common endocrine malignancy with an incidence that is increasing. ${ }^{1}$ In the United States alone an estimate of 48,020 new cases will be diagnosed in 2011, causing 1740 deaths. ${ }^{1}$ Differentiated thyroid cancer (DTC) and medullary thyroid cancer (MTC) which originate from follicular and neural crest $\mathrm{C}$ cells, respectively, are the most common types. Undifferentiated thyroid cancer is a highly aggressive and uncommon type of thyroid cancer. Most thyroid cancers are effectively treated with surgical resection, thyroid-stimulating hormone suppressive therapy, and ablation of the thyroid remnant with radioactive iodine (RAI). The prognosis is excellent with a 10 -year disease-related survival of $85 \%{ }^{2}$ However, patients with RAI-refractory and metastatic thyroid cancer have few therapeutic options, with response rates $<30 \%$ with the use of systemic chemotherapy, and usually short-lasting associated toxicities, and no proven survival benefit. ${ }^{3,4}$

Targeted therapies, anticancer agents developed to inhibit specific molecular pathways that drive various human tumors, have caused a paradigm shift in the treatment of solid and hematologic malignancies. The study of thyroid carcinomas has revealed somatic mutations in several pathways such as B-type Raf kinase (B-Raf), K-Ras, 
and rearranged during transfection (RET)/papillary thyroid carcinoma (PTC) that are associated with development and progression of approximately $70 \%$ of these malignancies. ${ }^{5}$ Therefore, lack of effective systemic therapies combined with a high incidence of driver mutations and overexpression of molecular pathways make refractory thyroid cancer an ideal candidate for treatment with novel targeted agents. Multikinase inhibitors (MKIs) have been shown to be the most promising targeted therapies against this disease by blocking pathway signaling like angiogenesis and, concomitantly, inhibiting mutated pathways (Table 1). The results of the ZETA (Zactima Efficacy in Thyroid Cancer Assessment) trial led the United States Food and Drug Administration to approve vandetanib for the treatment of symptomatic or progressive MTC, constituting this the first targeted agent approved for thyroid cancer. ${ }^{6}$ The molecular rationale for targeting thyroid cancer with these agents is discussed as well as the promising results of the treatment with MKIs in refractory thyroid carcinoma.

\section{Molecular pathways and therapeutic targets in thyroid cancer Raf/mitogen-activated protein kinase (MAPK) pathway}

The activation of the MAPK pathway plays a major role in the carcinogenesis of PTC, the most common thyroid malignancy. Raf, MAPK kinase, and MAPK are all serine/ threonine-selective protein kinases. The MAPK pathway is activated by growth factor-stimulated cell surface receptors such as epidermal growth factor receptor. ${ }^{7}$ When the cell surface receptor is phosphorylated, it activates Sos which consequently promotes Ras (a guanosine triphosphatase) to change its guanosine diphosphate for a guanosine triphosphate. Activated Ras activates the protein kinase activity of Raf kinase which phosphorylates and activates MAPK kinase. ${ }^{8}$
Finally, MAPK kinase phosphorylates and activates MAPK that, in turn, can now activate a transcription factor, such as myc. Among the three forms of Raf kinases, B-Raf is the most potent activator of the MAPK pathway. ${ }^{8}$ About $45 \%$ of sporadic PTC can have mutations in the $B$-Raf gene, making these the most common genetic alteration found in patients with thyroid cancer. ${ }^{9}$ More than $90 \%$ of $B$-Raf mutations exist in the V600E mutation (T1799A) in exon 15; abnormality is also present in $77.8 \%$ of patients with recurrent disease. ${ }^{9}$ B-Raf V600E mutation has been associated with several adverse pathologic prognostic features in PTC-like extrathyroidal invasion, multicentricity, presence of nodal metastases, and absence of tumor capsule. Moreover, it is associated with an increased rate of tumor recurrence and treatment failure. ${ }^{10,11}$

\section{Vascular endothelial growth factor (VEGF) pathway}

One of the major developments on anticancer therapy of the last two decades is the essential role of angiogenesis in tumor growth and metastasis; therefore, controlling tumorassociated angiogenesis is now a key tactic in limiting cancer progression. VEGF-A, the major mediator of tumor angiogenesis, is part of the VEGF family of structurally related molecules. VEGF-A promotes the proliferation and survival of endothelial cells and increases vascular permeability. ${ }^{12}$ VEGF-A signals through VEGF receptor 2 (VEGFR-2), the major VEGF signaling receptor that mediates sprouting angiogenesis. The binding of VEGF to VEGFR-2 leads to dimerization of the receptor, followed by intracellular activation of a cascade of different signaling pathways such as Raf/MAPK and phosphatidylinositol 3' kinase (PI3K)-Akt pathways. ${ }^{13}$ Both DTC and MTC have been found to express high levels of both angiopoietin-2 and VEGF and upregulation of its main receptor, VEGFR-2, with respect to normal thyroid. ${ }^{14,15}$ Moreover, increased expression of VEGF in

Table I Kinases inhibited by multikinase inhibitors with clinical activity in refractory thyroid cancer

\begin{tabular}{|c|c|c|c|c|c|c|c|c|c|}
\hline & VEGFR-2 & PDGFR & RET & EGFR & c-KIT & B-Raf & FLT3 & MET & BCR/ABL \\
\hline Pazopanib & $x$ & $x$ & & & $x$ & & & & \\
\hline Lenvatinib & $x$ & $x$ & $x$ & & $x$ & & & & \\
\hline Vandetanib & $x$ & & $x$ & $x$ & & & & & \\
\hline Motesanib & $x$ & $x$ & & & $x$ & & & & \\
\hline Sunitinib & $x$ & $x$ & $x$ & & $x$ & & $x$ & & \\
\hline Sorafenib & $x$ & $x$ & & & $x$ & $x$ & & & \\
\hline Axitinib & $x$ & $x$ & & & $x$ & & & & \\
\hline Cabozantinib & $x$ & & $x$ & & & & & $x$ & \\
\hline Imatinib & & & $x$ & & & & & & $x$ \\
\hline
\end{tabular}

Abbreviations: BCR/ABL, breakpoint cluster/Abelson; B-Raf, B-type Raf kinase; EGFR, epidermal growth factor receptor; FLT3, FMS-like tyrosine kinase receptor 3; PDGFR, platelet-derived growth factor receptor; RET, rearranged during transfection; VEGFR-2, vascular endothelial growth factor receptor-2. 
thyroid cancer has been associated with an increase in tumor size, local and distant metastasis, and poor prognosis. ${ }^{14,16}$

\section{RET pathway}

$R E T$ protooncogene is located in the chromosome 10q11.2. RET encodes a receptor tyrosine kinase that is expressed in neuroendocrine cells as well as thyroid $\mathrm{C}$ cells, adrenal medullary cells, and neural cells (including parasympathetic and sympathetic ganglion cells). The RET receptor consists of an extracellular portion, a transmembrane portion, and an intracellular portion, which contains two tyrosine kinase subdomains (TK1 and TK2) that are involved in the activation of several intracellular signal transduction pathways. In physiological conditions, activation of RET requires the formation of a multimeric complex with a coreceptor of the glycosylphosphatidylinositol-anchored glial cell line-derived neurotrophic factor family $\alpha$ coreceptors and one of their ligands, the glial cell line-derived neurotrophic factor family of ligands. ${ }^{17}$ The ligand binding leads to formation of the complex and RET dimerization, kinase activation, and signaling to the nucleus. Activation of RET has been shown to signal through multiple pathways, including Ras/ extracellular signal-related kinase pathway, PI3K, and Src, among others (Figure 1).
Activating mutations and translocation of RET are a common abnormality of both PTC and MTC. Translocations of $R E T$ are seen in $\leq 30 \%$ of PTC, and is commonly seen in cases of PTC associated with radiation exposure. ${ }^{18}$ Moreover, activating mutations are common in sporadic and hereditary MTC, leading to ligand-independent dimerization and phosphorylation. ${ }^{18}$

\section{MKIs in thyroid cancer Vandetanib}

Vandetanib is an orally bioavailable agent that is a multimarket kinase inhibitor that also has antiangiogenesis properties (targets VEGFR-2 and VEGFR-3, epidermal growth factor receptor, and RET kinase). ${ }^{19}$ It was found to be a promising agent for MTC because, besides its effects on angiogenesis, it has inhibitory effects in RET activation, a well-known protooncogene that is predominant in this disease. Two phase II trials have been reported so far using vandetanib in patients with MTC. The first was conducted in patients with locally advanced or metastatic hereditary MTC with RET germline mutation using vandetanib at $300 \mathrm{mg}$ orally per day. A total of 30 patients were accrued and partial response was reported in six patients $(20 \%)$ with stable disease in other nine subjects $(30 \%)$. Grade $1-2$ adverse

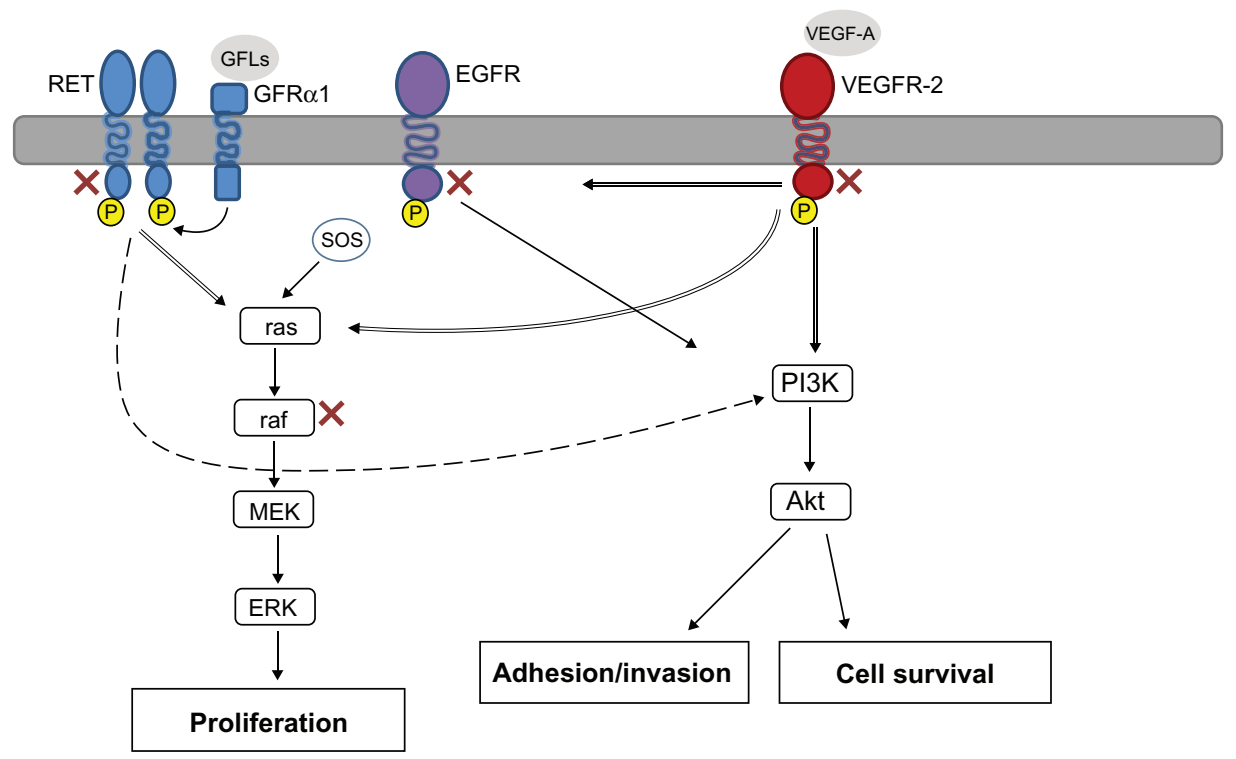

Figure I Molecular pathways targeted by multikinase inhibitors in refractory thyroid cancer.

Notes: Rearranged during transfection is the receptor for members of the glial cell line-derived neurotrophic factor family of extracellular signaling molecules or ligands. The complex of the glial cell line-derived neurotrophic factor family of ligands with the coreceptor glial cell line-derived neurotrophic factor family receptor $\alpha$ brings together two molecules of rearranged during transfection, triggering transautophosphorylation of specific tyrosine residues within the tyrosine kinase domain of each rearranged during transfection molecule. Rearranged during transfection can increase proliferation and survival through several pathways such as Ras/extracellular signal-related kinase and phosphatidylinositol 3' kinase. Both vascular endothelial growth factor-2 and epidermal growth factor pathways can also induce proliferation, invasion, and survival by activation of both Ras/extracellular signal-related kinase and phosphatidylinositol 3' kinase pathways. Marked in red are the targets inhibited by multikinase inhibitors.

Abbreviations: EGFR, epidermal growth factor; ERK, extracellular signal-regulated kinase; GFL, glial cell line-derived neurotrophic factor family of ligands; GFR $\alpha$, glial cell line-derived neurotrophic factor family $\alpha$ coreceptor; MAPK, mitogen-activated protein kinase; MEK, mitogen-activated protein kinase kinase; PI3K, phosphatidylinositol 3' kinase; RET, rearranged during transfection; VEGF-A, vascular endothelial growth factor A; VEGFR2, vascular endothelial growth factor receptor-2. 
events (presented in $>50 \%$ of the patients) were: rash, diarrhea, fatigue, and nausea. Grade 3 adverse events included asymptomatic QTc prolongation (17\%), rash, and diarrhea (both 10\%). ${ }^{20}$ The other phase II trial conducted by Robinson et al used vandetanib in a lower dose $(100 \mathrm{mg})$ in 19 patients, $79 \%$ of them with confirmed RET germline mutation. ${ }^{21}$ Postprogression dose increment to vandetanib $300 \mathrm{mg}$ was allowed in eligible patients. In a preliminary report with a median duration of treatment of 274 days, 14 patients remained on the $100 \mathrm{mg}$ dose and the dose after progression was raised to $300 \mathrm{mg}$ in two patients. From this group of 16 evaluable patients, partial responses were reported in two patients $(10 \%)$, stable disease for more than 6 months in six patients (31\%), and two other patients had disease progression.

The first phase III trial reported using a MKI was the ZETA (Zactima Efficacy in Thyroid Cancer Assessment) trial, a randomized, double-blind phase III trial in patients with locally advanced or metastatic thyroid cancer using $300 \mathrm{mg}$ daily of vandetanib versus placebo. ${ }^{22}$ The median age of the 331 patients included was 52 years; $56 \%$ had a positive RET mutation status. After a median follow-up of 24 months, a statistically significant progression-free survival (PFS), overall response rate, disease control rate, and biochemical response were observed for vandetanib versus placebo. This was the first phase III trial that demonstrated an improved PFS using an MKI in patients with thyroid cancer; thus, vandetanib was approved in April 2011 by the United States Food and Drug Administration for this indication. ${ }^{19}$ Recently, there have been attempts to combine biologic agents with chemotherapy or among themselves in several trials of head and neck and thyroid cancers. Vandetanib was recently combined with bortezomib, a proteasome inhibitor (approved for the treatment of multiple myeloma). ${ }^{23}$ The rationale of the trial was based on preclinical evidence which has shown that addition of bortezomib enhanced vandetanib activity against MTC cell lines. This was a phase I trial designed to assess the safety, tolerance, and activity of vandetanib plus bortezomib in 21 patients with MTC. A dose escalation schema with bortezomib (1-1.3 $\mathrm{mg} / \mathrm{m}^{2}$ ) intravenously on days one, four, eight, and eleven and vandetanib (100-300 mg) orally daily (28-day cycles) was in place. Grade 3 toxicities were: hypertension $(24 \%)$, fatigue (19\%), thrombocytopenia $(10 \%)$, diarrhea $(10 \%)$, and arthralgia (10\%), with keratoacanthoma, hyperkalemia, pulmonary hemorrhage, edema, and prolonged QTc each in one patient (5\%). There was one dose limiting toxicity - grade 3 thrombocytopenia at a bortezomib/vandetanib dose of 1.3/200 in cycle two, but no grade 4-5 toxicities. The 17 patients who had MTC were metastatic.
Median calcitonin and carcinoembryonic antigen levels were 5289 and 113, respectively. Five (29\%) patients had partial response and eight (47\%) attained stable disease. Six patients who attained stable disease maintained their disease control for more than 6 months. The maximum tolerated dose for the combination was bortezomib $1.3 \mathrm{mg} / \mathrm{m}^{2}$ intravenously on days one, four, eight, and eleven and vandetanib at $300 \mathrm{mg}$ orally daily. ${ }^{23}$

\section{Pazopanib}

Pazopanib is a small-molecule inhibitor of VEGFR-1, VEGFR-2, and VEGFR-3, platelet-derived growth factor receptor- $\beta$ (PDGFR- $\beta$ ), and c-Kit, and is currently approved for the treatment of renal cell carcinoma. ${ }^{24} \mathrm{~A}$ phase II trial using pazopanib at $800 \mathrm{mg}$ daily enrolled 39 patients with metastatic, rapidly progressive, RAI-refractory DTC. ${ }^{25}$ Of the 37 evaluable patients, confirmed partial responses were recorded in 18 patients (49\%) and the likelihood of response lasting longer than 1 year was calculated to be $66 \%$. Interestingly, the maximum pazopanib plasma concentration correlated with the maximum change in tumor size and was significantly higher in the 18 patients who achieved confirmed partial response by Response Evaluation Criteria in Solid Tumors criteria when compared to those who did not. Dose reduction was required in $16(43 \%)$ of 37 patients because of adverse effects. ${ }^{25} \mathrm{~A} \geq 30 \%$ decrease in thyroglobulin concentrations was observed in $88 \%$ of the patients with data available. The most frequent adverse events that required a dose reduction were fatigue, skin and hair hypopigmentation, diarrhea, and nausea. The death of two patients during treatment was attributed to underlying disorders.

\section{Lenvatinib (E7080)}

Lenvatinib is an orally administered MKI with a wide therapeutic range targeting several important kinases in thyroid cancers such as VEGFR-1, VEGFR-2, VEGFR-3, fibroblast growth factor receptor-1 (FGFR-1), FGFR-2, FGFR-3, FGFR-4, PDGFR- $\beta$, RET, and c-Kit. It also has the benefit of not prolonging the QTc interval like other MKIs. ${ }^{26}$ A phase II study with lenvatinib $24 \mathrm{mg}$ once daily until disease progression was conducted in 58 patients with advanced RAI-refractory DTC whose disease had progressed during the prior 12 months. ${ }^{27}$ Confirmed partial responses were observed in 29 patients $(50 \%)$ based on investigator assessment, with $65 \%$ of the responses seen at 8 weeks. Interestingly, the response rate for patients who received prior VEGFR-directed therapy was $41 \%$. Of the patients enrolled, $35 \%$ required dose reduction for management of toxicity, 
and $23 \%$ were withdrawn from therapy due to toxicity. The most common adverse events were hypertension, diarrhea, decreased appetite, weight loss, and proteinuria; all them had an incidence of $<10 \%$ for grade 3 . Five patients $(8.6 \%)$ experienced grade 4 events. ${ }^{27}$

\section{Sorafenib}

Three phase II clinical trials using sorafenib in patients with metastatic RAI-refractory thyroid carcinoma have been published to date. The first trial was conducted by Kloos et al in which 56 patients were enrolled; partial response was seen in six of the 41 patients with PTC included; stable disease lasted $>6$ months in 23 patients. ${ }^{28}$ The median duration of partial response was 7.5 months and median PFS was 15 months. Grade 3 adverse events included hand-foot skin reaction, musculoskeletal pain, and fatigue. Interestingly, a high incidence of the $B$-Raf mutation was found in 17 of the 22 PTCs $(77 \%)$ analyzed, with 14 of these mutations being V600E, whereas three other patients had a K601E mutation. No patients with MTC were included and no partial responses were reported in non-PTC patients. The second phase II trial was conducted by Gupta-Abramson et al in 30 patients who were treated with sorafenib $400 \mathrm{mg}$ orally twice daily. ${ }^{29}$ Seven patients had partial response lasting 18-84 weeks, and 16 patients had stable disease lasting 14-89 weeks; median PFS was 79 weeks. Of note, $95 \%$ patients for whom serial thyroglobulin levels were available showed a decrease in thyroglobulin levels, with a mean decrease of $70 \%$. In terms of toxicity, a single patient died of liver failure that was likely to be treatment-related. Although the presence of the $B$-Raf mutation has been related with poor outcome and the results of these trials are encouraging, the correlation between the presence of $B$-Raf V600E mutation and clinical response to sorafenib has yet to be elucidated. Preliminary results of another open-label phase II study of sorafenib in 55 patients with metastatic, RAI-refractory thyroid carcinoma conducted by Brose et al reported an increased PFS for patients with $B-R a f \mathrm{~V} 600 \mathrm{E}$, compared with that of patients with wild-type $B$-Raf ( 84 versus 54 weeks; $P=0.028) .{ }^{30}$ More recently, another phase II trial by Lam et al looking at patients with advanced MTC was published. Herein, patients were stratified into two different groups: hereditary (arm A) or sporadic (arm B); all patients received sorafenib $400 \mathrm{mg}$ orally twice daily. ${ }^{31}$ Sixteen patients were enrolled in arm B, and arm A was closed due to a slow accrual of patients with sporadic MTC. One patient achieved partial response (6.3\%) and 14 attained stable disease $(87.5 \%)$, with four of them having stable disease lasting $>15$ months. The median PFS for arm B was 17.9 months. $^{31}$

\section{Motesanib}

Rosen et al initially reported partial responses in three of seven patients with thyroid cancer enrolled in a phase I study of motesanib in patients with advanced solid tumors. ${ }^{32}$ Two phase II trials using $125 \mathrm{mg}$ of motesanib diphosphate orally once daily have been conducted in patients with advanced or metastatic RAI-refractory thyroid cancer. The first trial by Sherman et al treated 93 patients with DTC; 57 (61\%) of them were PTC. ${ }^{33}$ The overall response rate was $14 \%$ and stable disease was achieved in $67 \%$ of the patients and maintained for $\geq 24$ weeks in $35 \%$ of the patients; $8 \%$ had progression of disease. The median duration of response was 32 weeks and median PFS was 40 weeks. Diarrhea (59\%), hypertension $(56 \%)$, fatigue (46\%), and weight loss (40\%) were the most common treatment-related adverse events. The second phase II trial by Schlumberger et al also used motesanib at $125 \mathrm{mg}$ daily, but in 91 patients with MTC. Efficacy was somewhat lower than that in the previous study. ${ }^{34}$ Response rate was characterized by partial remission in two patients ( $2 \%$ ), stable disease in $81 \%$, and a median PFS of 48 weeks. Decrease in serum calcitonin and carcinoembryonic antigen during treatment was seen in $83 \%$ and $75 \%$ of the patients, respectively. The most common treatment-related adverse events were similar to other trials with motesanib including diarrhea, fatigue, hypertension, and anorexia. Hypothyroidism was reported to be considerably worse in $29 \%$ of the patients. Circulating biomarkers of angiogenesis and apoptosis were obtained in patients participating in these last two trials. Samples were collected at baseline and up to 4 weeks after the end of the study. ${ }^{35}$ Levels of soluble VEGFR-1, placental growth factor (PlGF), VEGF, and basic FGF were measured. Results of these biomarkers revealed changes in serum PlGF as early as 1 week into treatment which, in fact, correlated with best tumor response. Those patients who had 4.7-fold increase or greater in PlGF levels after 1 week of treatment were more likely to achieve partial remission than those who reached levels less than the 4.7 cutoff. The response rate among patients with a greater than 4.7-fold increase in PIGF was 30\% compared with 3\% for those below. There was also a significant separation between responders and nonresponders at a 1.6-fold decrease in VEGFR-2 after 3 weeks of treatment Baseline serum VEGF levels $<671 \mathrm{pg} / \mathrm{mL}$ correlated with significantly longer PFS. ${ }^{35}$

\section{Sunitinib}

Three phase II trials with sunitinib are reported here. The first trial used sunitinib $50 \mathrm{mg}$ daily on a 4-week-on/2-week-off schedule, and included 43 subjects with MTC and DTC. ${ }^{36}$ 
The overall response rate in the 31 patients with DTC was $13 \%$, with stable disease in $68 \%$ of them. On the other hand, no responses were observed in patients with MTC, although stable disease was observed in the majority of patients (83\%). ${ }^{36}$ Grade 3-4 adverse events included neutropenia, thrombocytopenia, hypertension, fatigue, palmar-plantar erythrodysesthesia, and gastrointestinal symptoms. In the second trial, sunitinib was given at $37.5 \mathrm{mg}$ daily to 2-deoxy2-[18F]fluoro-D-glucose positron emission tomography-avid advanced thyroid cancers. Three patients had MTC and 15 patients had DTC; the 2-deoxy-2-[18F]fluoro-D-glucose positron emission tomography response rate was observed in seven patients (all of them with DTC histology). The most common grade 3 adverse event was neutropenia (28\%); no grade 4 toxicities were reported. ${ }^{37}$ Another phase II study by Ravaud et al also administered sunitinib at $50 \mathrm{mg} /$ day for 4 weeks every 6 weeks. ${ }^{38}$ Patients had anaplastic, MTC, or RAI-refractory DTC which had being progressing during the 6 months prior to study start. Seventeen patients were enrolled. Median age was 64.3 years old, metastatic sites included lung, lymph nodes, and bones, the treatment was well tolerated with the main side effects including hypertension, asthenia, mucositis, hand-foot syndrome, and thrombocytopenia. Fifteen subjects were evaluable for response; of those, one patient had evidence of partial remission and 12 patients attained stable disease, with one patient showing $>90 \%$ decrease of thyroglobulin and another patient with a dramatic decrease of symptoms.

\section{Axitinib}

Axitinib (AG-013736) is an oral, potent, and selective small molecule inhibitor of VEGFR-1, VEGFR-2, and VEGFR-3, which was recently approved for the treatment of metastatic kidney cancer. It has inhibitory properties at picomolar concentrations against VEGFR-1, VEGFR-2, and VEGFR3 , and at nanomolar concentrations against PDGFR- $\beta$ and c-Kit. This agent has been shown to affect VEGF-dependent fenestrations and cause regression of tumor vessels. ${ }^{39-42}$ The dose of axitinib was determined after a phase I trial in which 36 patients with solid tumors were administered this novel agent. ${ }^{43}$ The recommended dose is $5 \mathrm{mg}$ orally twice daily. Axitinib demonstrated activity against all histologic subtypes of thyroid cancer and the main side effect was hypertension, which was easily managed with antihypertensives.

A phase II trial evaluated the efficacy of axitinib in patients with a diagnosis of RAI-refractory thyroid carcinoma. A total of 60 patients were enrolled in this study. ${ }^{44}$ Patients had all histologic subtypes of thyroid cancer and they were treated with $5 \mathrm{mg}$ twice daily. All histologic subtypes achieved a clinical response, with eight partial responses in patients with papillary histology, six in follicular, two in medullary, and one in anaplastic thyroid carcinoma. A total of $23(38 \%)$ patients achieved stable disease and there was a median PFS of 18.1 months. A total of 19 (32\%) patients reported at least one grade 3 adverse effect from axitinib. The most common adverse effect was hypertension and there were three associated grade 4 adverse effects, which included cerebrovascular accident, hypertension, and reversible leukoencephalopathy. Finally, a total of eight (13\%) patients had to discontinue treatment due to adverse effects. ${ }^{44}$

\section{Cabozantinib}

Cabozantinib (XL184) is a dual inhibitor, exerting its effects over MET and VEGF pathways. ${ }^{45,46}$ Cabozantinib is a small molecule tyrosine kinase inhibitor that targets multiple receptors such as VEGFR-1, VEGFR-2, c-Met, RET, c-Kit, FMS-like tyrosine kinase receptor 3, Tie-2, and RET/PTC. The recommended dose for cabozantinib was determined at $175 \mathrm{mg}$ daily orally after a phase I trial. ${ }^{47}$ In this study, eight of 16 patients with MTC achieved partial response and all other patients achieved stable disease. Adverse effects included diarrhea, nausea, fatigue, mucosal inflammation, anorexia, elevated liver enzymes, hypertension, and vomiting. Further studies are on their way to study this promising novel agent.

\section{Imatinib}

Imatinib is currently approved by the United States Food and Drug Administration and the European Medicines Agency for the treatment of chronic myelogenous leukemia, dermatofibrosarcoma protuberans, and gastrointestinal stromal tumor. ${ }^{48-52}$ Imatinib targets the BCR-ABL gene translocation, PDGFR, stem cell factor, and c-Kit, and causes inhibition of proliferation and apoptosis in cells that express them. ${ }^{53}$ Due to RET overexpression in thyroid carcinoma and the fact that both RET and c-Kit are part of the same subfamily of tyrosine kinase receptors, multiple attempts have been made to exploit the use of imatinib in thyroid carcinomas. A phase II trial treated 15 patients with imatinib at $600 \mathrm{mg}$ daily and in case of clinical response the dose could be escalated to $800 \mathrm{mg}$ daily. ${ }^{54}$ All patients enrolled in this trial had a confirmed diagnosis of MTC. There were no objective responses observed and the median duration of treatment was 4 months. A similar trial enrolled nine patients with diagnosis of MTC. The patients were treated with imatinib $600 \mathrm{mg}$ daily with a median duration of 13 months. A total of seven patients 
Table 2 Phase II and III trials reported of multikinase inhibitors in patients with advanced and refractory thyroid cancer

\begin{tabular}{|c|c|c|c|c|c|c|}
\hline Agents & Trial & Subtypes included & $\begin{array}{l}\text { Number } \\
\text { of patients }\end{array}$ & $\begin{array}{l}\text { PR } \\
\% \text { (n) }\end{array}$ & $\begin{array}{l}\text { SD } \\
\%(n)\end{array}$ & $\begin{array}{l}\text { PFS } \\
\text { (months) }\end{array}$ \\
\hline \multirow[t]{3}{*}{ Vandetanib } & ZETA trial ${ }^{22, *}$ & MTC & 331 & 45 & 42 & $30.5^{* *}$ \\
\hline & Wells et $a^{20}$ & MTC & 30 & $20(6)$ & $30(9)$ & NR \\
\hline & Robinson et $\mathrm{a}^{21}$ & MTC & 19 & $10(2)$ & $31(6)$ & NR \\
\hline \multirow[t]{2}{*}{ Motesanib } & Schlumberger et $\mathrm{al}^{34}$ & MTC & 91 & $2(2)$ & $81(74)$ & II \\
\hline & Sherman et $\mathrm{al}^{33}$ & DTC & 93 & $14(13)$ & $67(62)$ & 9 \\
\hline \multirow[t]{2}{*}{ Sorafenib } & Kloos et $\mathrm{al}^{28}$ & DTC & 56 & $11(6)$ & $56(3 I)$ & 15 \\
\hline & Gupta-Abramson et al ${ }^{29}$ & DTC, MTC & 30 & $23(7)$ & $53(16)$ & 18 \\
\hline Axitinib & Cohen et $\mathrm{al}^{44}$ & DTC, MTC, ATC & 60 & $18(11)$ & $23(14)$ & I8.1 \\
\hline \multirow[t]{2}{*}{ Sunitinib } & Cohen et $\mathrm{al}^{36}$ & DTC, MTC & 43 & $13(6)$ & $68(30)$ & NR \\
\hline & Goulart et a ${ }^{37}$ & DTC, MTC & 18 & $38(7)$ & & \\
\hline Cabozantinib & Kurzrock et $\mathrm{al}^{47, \dagger}$ & MTC & 22 & $50(11)$ & $50(\mathrm{II})$ & NR \\
\hline Pazopanib & Bible et $\mathrm{a}^{25}$ & DTC & 32 & $19(6)$ & $69(22)$ & NR \\
\hline \multirow[t]{2}{*}{ Imatinib } & de Groot et $\mathrm{al}^{54}$ & MTC & 15 & 0 & $26(4)$ & NR \\
\hline & Frank-Raue et al ${ }^{55}$ & MTC & 9 & 0 & $77(7)$ & NR \\
\hline
\end{tabular}

Notes: *Only phase III trial reported; **estimated; ${ }^{\dagger}$ phase I trial with a subgroup of patients with medullary thyroid cancer.

Abbreviations: ATC, anaplastic thyroid cancer; DTC, differentiated thyroid cancer; MTC, medullary thyroid cancer; NR, not reported; PFS, progression-free survival; PR, partial response; SD, stable disease; ZETA, Zactima Efficacy in Thyroid Cancer Assessment.

achieved stable disease at 3 months, but only one of these remained with stable disease at 12 months. There were no clinical responses, and the median PFS was 6 months. ${ }^{55}$

\section{Conclusion}

Systemic chemotherapy has been used for years for advanced or metastatic thyroid carcinomas with low efficacy. Angiogenesis plays a crucial role in tumor progression because tumor angiogenesis is driven by host-derived circulating factors. Nowadays, there are multiple novel therapies that target angiogenesis in clinical trials. The most successful agents target the VEGFRs, with potential targets including the mutant kinases associated with papillary and medullary oncogenesis. VEGFR inhibition has relied on the use of small molecular inhibitors. These drugs inhibit tyrosine kinase activity of VEGFRs as well as other tyrosine kinases. At least one phase III trial has showed improvements in PFS with the use of vandetanib, an MKI of VEGFR, epidermal growth factor receptor, and RET.

As discussed in this manuscript, not all MKIs have the same effect on RAI-refractory thyroid cancers. Some are more effective on certain histologic subtypes (Table 2). Thus, it is important to identify biomarkers which can help sort out the best therapeutic choice. Efforts in this regard are underway in clinical trials. As an example, levels of soluble VEGFR-1, PlGF, VEGF, and basic FGF have been studied as biomarkers with the use of motesanib. Initial analyses have shown that their levels may correlate with response and/or PFS. Randomized trials for other agents are currently underway, and the treatment for patients with metastatic or advanced thyroid carcinoma now emphasizes clinical trial opportunities for novel agents with considerable promise.

\section{Disclosure}

Luis Raez has received research support from Pfizer. All other authors report no conflicts of interest in this work.

\section{References}

1. Siegel R, Ward E, Brawley O, Jemal A. Cancer statistics, 2011: the impact of eliminating socioeconomic and racial disparities on premature cancer deaths. CA Cancer J Clin. 2011;61(4):212-236.

2. Eustatia-Rutten CF, Corssmit EP, Biermasz NR, Pereira AM, Romijn JA, Smit JW. Survival and death causes in differentiated thyroid carcinoma. J Clin Endocrinol Metab. 2006;91(1):313-319.

3. Gottlieb JA, Hill CS Jr. Chemotherapy of thyroid cancer with adriamycin. Experience with 30 patients. N Engl J Med. 1974;290(4):193-197.

4. Shimaoka K, Schoenfeld D, DeWys WD, Creech RH, DeConti R. A randomized trial of doxorubicin versus doxorubicin plus cisplatin in patients with advanced thyroid carcinoma. Cancer. 1985;56(9):2155-2160.

5. Nikiforov YE, Ohori NP, Hodak SP, et al. Impact of mutational testing on the diagnosis and management of patients with cytologically indeterminate thyroid nodules: a prospective analysis of 1056 FNA samples. J Clin Endocrinol Metab. 2011;96(11):3390-3397.

6. Commander H, Whiteside G, Perry C. Vandetanib: first global approval. Drugs. 2011;71(10):1355-1365.

7. Roberts PJ, Der CJ. Targeting the Raf-MEK-ERK mitogen-activated protein kinase cascade for the treatment of cancer. Oncogene. 2007;26(22):3291-3310.

8. Xing M. BRAF mutation in thyroid cancer. Endocr Relat Cancer. 2005;12(2):245-262.

9. Henderson YC, Shellenberger TD, Williams MD, et al. High rate of BRAF and RET/PTC dual mutations associated with recurrent papillary thyroid carcinoma. Clin Cancer Res. 2009;15(2):485-491.

10. Lupi C, Giannini R, Ugolini C, et al. Association of BRAF V600E mutation with poor clinicopathological outcomes in 500 consecutive cases of papillary thyroid carcinoma. J Clin Endocrinol Metab. 2007;92(11):4085-4090. 
11. Xing M, Westra WH, Tufano RP, et al. BRAF mutation predicts a poorer clinical prognosis for papillary thyroid cancer. J Clin Endocrinol Metab. 2005;90(12):6373-6379.

12. Weis SM, Cheresh DA. Tumor angiogenesis: molecular pathways and therapeutic targets. Nat Med. 2011;17(11):1359-1370.

13. Kerbel RS. Tumor angiogenesis. N Engl J Med. 2008;358(19): 2039-2049.

14. Bunone G, Vigneri P, Mariani L, et al. Expression of angiogenesis stimulators and inhibitors in human thyroid tumors and correlation with clinical pathological features. Am J Pathol. 1999;155(6): 1967-1976.

15. Klein M, Picard E, Vignaud JM, et al. Vascular endothelial growth factor gene and protein: strong expression in thyroiditis and thyroid carcinoma. J Endocrinol. 1999;161(1):41-49.

16. Lennard CM, Patel A, Wilson J, et al. Intensity of vascular endothelial growth factor expression is associated with increased risk of recurrence and decreased disease-free survival in papillary thyroid cancer. Surgery. 2001;129(5):552-558.

17. Mologni L. Development of RET kinase inhibitors for targeted cancer therapy. Curr Med Chem. 2011;18(2):162-175.

18. Houvras Y. Completing the arc: targeted inhibition of RET in medullary thyroid cancer. J Clin Oncol. 2012;30(2):200-202.

19. Langmuir PB, Yver A. Clin Pharmacol Ther. 2012 Jan;91(1):71-80

20. Wells SA Jr, Gosnell JE, Gagel RF, et al. Vandetanib for the treatment of patients with locally advanced or metastatic hereditary medullary thyroid cancer. J Clin Oncol. 2010;28(5):767-772.

21. Robinson BG, Paz-Ares L, Krebs A, Vasselli J, Haddad R. Vandetanib $(100 \mathrm{mg})$ in patients with locally advanced or metastatic hereditary medullary thyroid cancer. J Clin Endocrinol Metab. 2010;95(6):2664-2671.

22. Wells SA Jr, Robinson BG, Gagel RF, et al. Vandetanib in patients with locally advanced or metastatic medullary thyroid cancer: a randomized, double-blind phase III trial. J Clin Oncol. 2012;30(2):134-141.

23. Gramza AW, Wells SA, Balasubramaniam S, Fojo AT. Phase I/II trial of vandetanib and bortezomib in adults with locally advanced or metastatic medullary thyroid cancer: phase I results [abstract]. $J$ Clin Oncol. 2011;29 Suppl:5565.

24. Sternberg CN, Davis ID, Mardiak J, et al. Pazopanib in locally advanced or metastatic renal cell carcinoma: results of a randomized phase III trial. J Clin Oncol. 2010;28(6):1061-1068.

25. Bible KC, Suman VJ, Molina JR, et al. Efficacy of pazopanib in progressive, radioiodine-refractory, metastatic differentiated thyroid cancers: results of a phase 2 consortium study. Lancet Oncol. 2010;11(10):962-972.

26. Sherman SI, Jarzab B, Cabanillas ME, et al. A phase II trial of the multitargeted kinase inhibitor E7080 in advanced radioiodine (RAI)refractory differentiated thyroid cancer (DTC) [abstract]. J Clin Oncol. 2011;29 Suppl:5503.

27. Shumaker R, Zhou M, Ren M, Fan J, Martinez G, Darpo B. Lenvatinib (E7080) does not prolong the QTc interval: results from a thorough QT study in healthy volunteers [abstract]. Mol Cancer Ther. 2011; 10(11 Suppl 1):C116.

28. Kloos RT, Ringel MD, Knopp MV, et al. Phase II trial of sorafenib in metastatic thyroid cancer. J Clin Oncol. 2009;27(10):1675-1684.

29. Gupta-Abramson V, Troxel AB, Nellore A, et al. Phase II trial of sorafenib in advanced thyroid cancer. J Clin Oncol. 2008;26(29):4714-4719.

30. Brose MS, Troxel AB, Redlinger M, et al. Effect of BRAFV600E on response to sorafenib in advanced thyroid cancer patients [abstract]. J Clin Oncol. 2009;27(Suppl 15):6002.

31. Lam ET, Ringel MD, Kloos RT, et al. Phase II clinical trial of sorafenib in metastatic medullary thyroid cancer. J Clin Oncol. 2010;28(14): 2323-2330.

32. Rosen LS, Kurzrock R, Mulay M, et al. Safety, pharmacokinetics, and efficacy of AMG 706, an oral multikinase inhibitor, in patients with advanced solid tumors. J Clin Oncol. 2007;25(17):2369-2376.

33. Sherman SI, Wirth LJ, Droz JP, et al. Motesanib diphosphate in progressive differentiated thyroid cancer. N Engl J Med. 2008;359(1):31-42.
34. Schlumberger MJ, Elisei R, Bastholt L, et al. Phase II study of safety and efficacy of motesanib in patients with progressive or symptomatic, advanced or metastatic medullary thyroid cancer. J Clin Oncol. 2009;27(23):3794-3801.

35. Bass MB, Sherman SI, Schlumberger MJ, et al. Biomarkers as predictors of response to treatment with motesanib in patients with progressive advanced thyroid cancer. $J$ Clin Endocrinol Metab. 2010;95(11):5018-5027.

36. Cohen EE, Needles BM, Cullen KJ, et al. Phase 2 study of sunitinib in refractory thyroid cancer [abstract]. J Clin Oncol. 2008;26 Suppl:6025.

37. Goulart B, Carr L, Martins RG, et al. Phase II study of sunitinib in iodine refractory, well-differentiated thyroid cancer (WDTC) and metastatic medullary thyroid carcinoma (MTC) [abstract]. J Clin Oncol. 2008;26 Suppl:6062.

38. Ravaud A, de la Fouchardiere C, Courbon F, et al. Sunitinib in patients with refractory advanced thyroid cancer: the THYSU phase II trial [abstract]. J Clin Oncol. 2008;26 Suppl:6058.

39. Inai $T$, Mancuso $M$, Hashizume $H$, et al. Inhibition of vascular endothelial growth factor (VEGF) signaling in cancer causes loss of endothelial fenestrations, regression of tumor vessels, and appearance of basement membrane ghosts. Am J Pathol. 2004;165(1):35-52.

40. Mancuso MR, Davias R, Norberg SM, et al. Rapid vascular regrowth in tumors after reversal of VEGF inhibition. $J$ Clin Invest. 2006;116(10):2610-2621

41. Kamba T, Tam BY, Hashizume H, et al. VEGF-dependent plasticity of fenestrated capillaries in the normal adult microvasculature. $\mathrm{Am} \mathrm{J}$ Physiol Heart Circ Physiol. 2006;290(2):H560-H576.

42. Baffert F, Le T, Sennino B, et al. Cellular changes in normal blood capillaries undergoing regression after inhibition of VEGF signaling. Am J Physiol Heart Circ Physiol. 2006;290(2):H547-H559.

43. Rugo HS, Herbst RS, Liu G, et al. Phase I trial of the oral antiangiogenesis agent AG-013736 in patients with advanced solid tumors: pharmacokinetics and clinical results. J Clin Oncol. 2005;23(24):5474-5483.

44. Cohen EE, Rosen LS, Vokes EE, et al. Axitinib is an active treatment for all histologic subtypes of advanced thyroid cancer: results from a phase II study. J Clin Oncol. 2008;26(29):4708-4713.

45. Joly AH. Simultaneous blockade of VEGF and HGF receptors results in potent anti-angiogenic and anti-tumor effects. Eur J Cancer. 2006; Suppl 4:35.

46. Sennino B, Naylor RM, Tabruyn SP, You WK, Aftab DT, McDonald DM. Reduction of tumor invasiveness and metastasis and prolongation of survival of RIP-Tag2 mice after inhibition of VEGFR plus c-Met by XL184 [abstract]. Mol Cancer Ther. 2011;8(12 Suppl 1):A13.

47. Kurzrock R, Sherman S, Hong DS, et al. A phase I study of XL184, a RET, VEGFR2, and MET kinase inhibitor, in patients (pts) with advanced malignancies, including a subgroup of pts with medullary thyroid cancer (MTC). Paper presented at: AACR-NCI-EORTC International Conference on Molecular Targets and Cancer Therapeutics; October 21-24, 2008; Geneva, Switzerland.

48. Druker BJ, Talpaz M, Resta DJ, et al. Efficacy and safety of a specific inhibitor of the BCR-ABL tyrosine kinase in chronic myeloid leukemia. N Engl J Med. 2001;344(14):1031-1037.

49. Demetri GD, von Mehren M, Blanke CD, et al. Efficacy and safety of imatinib mesylate in advanced gastrointestinal tumors. $N$ Engl J Med. 2002;347(7):472-480.

50. O'Brien SG, Guilhot F, Larson RA, et al. Imatinib compared with interferon and low-dose cytarabine for newly diagnosed chronic-phase chronic myelogenous leukemia. N Engl J Med. 2003;348(11):994-1004.

51. Verweij J, van Oosterom A, Blay JY, et al. Imatinib mesylate (STI-571 Glivec, Gleevec) is an active agent for gastrointestinal stromal tumours, but does not yield responses in other soft-tissue sarcomas that are unselected for this molecular target. Results from the EORTC Soft Tissue and Bone Sarcoma Group phase II study. Eur J Cancer. 2003;39(14):2006-2011.

52. Sawyers CL. Imatinib GIST keeps finding new indications: successful treatment of dermatofibrosarcoma protuberans by targeted inhibition of the platelet-derived growth factor receptor. J Clin Oncol. 2002;20(17):3568-3569. 
53. Druker BJ, Tamura S, Buchdunger E, et al. Effects of a selective inhibitor of the Abl tyrosine kinase on growth of Bcr-Abl positive cells. Nat Med. 1996;2(5):561-566.

54. de Groot JWB, Zonnenberg BA, Quarles van Ufford-Mannesse P, et al. A phase II trial of imatinib therapy for metastatic medullary thyroid carcinoma. J Clin Endocrinol Metab. 2007;92(9):3466-3469.
55. Frank-Raue K, Fabel M, Delorme S, Haberkorn U, Raue F. Efficacy of imatinib mesylate in advanced medullary thyroid carcinoma. Eur $J$ Endocrinol. 2007;157(2):215-220.

\section{Publish your work in this journal}

Biologics: Targets \& Therapy is an international, peer-reviewed journal focusing on the patho-physiological rationale for and clinical application of Biologic agents in the management of autoimmune diseases, cancers or other pathologies where a molecular target can be identified. This journal is indexed on PubMed Central, CAS, EMBase, Scopus

\section{Dovepress}

and the Elsevier Bibliographic databases. The manuscript management system is completely online and includes a very quick and fair peerreview system, which is all easy to use. Visit http://www.dovepress. com/testimonials.php to read real quotes from published authors. 